\title{
The role of imageability and frequency in language production and comprehension in first-episode and early-course psychosis
}

\author{
Iva Kužina' • Vlasta Erdeljac' • Martina Sekulić Sović $•$ Ninoslav Mimica ${ }^{2,3}$ \\ - Draženka Ostojićzi, • Aleksandar Savić2,3 \\ 'University of Zagreb, Faculty of Humanities and Social Sciences, Department of Linguistics, \\ ${ }^{2}$ University Psychiatric Hospital Vrapče, ${ }^{3}$ University of Zagreb, School of Medicine, Chair of Psychiatry \\ and Psychological Medicine, and 4 University of Zagreb, Faculty of Law, Social Work Study Centre
}

https://doi.org/10.17234/9789531758314.07

\begin{abstract}
This study is an overview of language production and the comprehension of associations depending on the lexical-semantic imageability and frequency features in subjects with first-episode and early-course psychosis. Concepts with low values of lexical-semantic feature imageability, unlike those with high imageability, demand greater cognitive effort in the creation of their mental image and their activation in the semantic memory. This study included two tests, a forced-choice language comprehension test and an elicited association production test, which were constructed for the purpose of the study on the basis of an existing database of psycholinguistic lexical-semantic features (Erdeljac et al., 2018). Twelve subjects diagnosed with first-episode early-course psychosis were recruited, as well as 12 healthy subjects as a control group. It was confirmed that the target group, the subjects with first-episode and early-course psychosis, processes language differently with regards to the imageability feature in the production test. The patient group had longer reaction times on both imageability conditions in relation to the control group, but while the control group had shorter reaction times on highly imageable words, the patient group's reaction times did not differ on account of the imageability condition. In the comprehension test, the patient group had overall longer reaction times independently of the imageability condition. Furthermore, in the comprehension test, both groups statistically more often chose associations that were of high and medium frequency, when compared to low frequency words, and the target group had longer reaction times on high- and medium-frequency words than on low-frequency words. The results analysis was conducted according to current psycholinguistic theories and further validate the theory that subjects with schizophrenia have increased activation and decreased inhibition in the spreading of activation in the semantic memory during language processing.
\end{abstract}

Key words: associations, imageability, frequency, early-onset psychosis, forced-choice comprehension test, associations production test 


\section{Introduction}

From a psycholinguistic viewpoint, psychosis, and hence schizophrenia, has most often been studied in terms of deficits in language production and comprehension related to their atypical production of associations. Idiosyncratic and loosely related associations are thought to be prevalent in language production in schizophrenia (Maher et al., 2005, Elvevåg et al., 2007). The basis of such production is thought to be an abnormal activity associated with intralexical automatic spreading of activation in the semantic memory in semantic processing at short SOA intervals (less than $300 \mathrm{~ms}$ ) which do not include extralexical strategic processes (Mathalon et al., 2010, Neely \& Keefe, 1989). Such abnormal activity has been described as a hyperactivity of spreading activation in the semantic memory (Spitzer et al., 1993; Ballerini, 2016) or a slowing of movements between different concepts in the semantic memory (Bokat \& Goldberg, 2003). In theories which support the first standpoint, semantic memory is conceptualized as a connectionist model in which each concept is represented as a node of links which are connections to other concepts and present their relations (according to their association strength, features, frequency of use, etc.). In search-and-retrieval processes in language processing, each concept is activated by the activation of its connections. A greater connections network is thought to increase the activation. In schizophrenia, and possibly psychosis in general, this activation, according to the theory of increased spreading of activation, is increased. Increased activation needs a stronger inhibition of incorrectly activated concepts, but it is thought to be decreased in schizophrenia. Based on a priming study, Spitzer et al. (1993) suggest that greater activation in the semantic memory needs a higher amount of inhibition, which is decreased in schizophrenia, and that results in slower reaction times in subjects with schizophrenia than in healthy control subjects. Citing an ERP study on indirect priming, Kreher et al. (2008) have concluded that subjects with schizophrenia display higher automatic activation and decreased inhibition of false answers. The question has arisen as to the influence of different lexical-semantic features, such as imageability and frequency, on language processing in subjects with first-episode and early-course psychosis, as they are considered to be among the lexical-semantic features that strongly influence language processing because the define the quantity and quality of the connections representing a concept.

The semantic memory is a store of knowledge about concepts which are used and manipulated during language processing. It is generally conceptualized as a network structure of nodes that represent concepts and are connected by relational links that are marked according to the features they represent (Minzenberg et al., 2002). The quantity and quality of connections representing a concept 
and linking it to other concepts depend on its features, such as frequency of use, concreteness, imageability, etc.

Imageability is the ability with which words activate their mental images. Highly imageable words require less cognitive effort in concept activation than do low-imageability words. In a connectionist-type framework, de Groot (1989) concludes that highly imageable and concrete words have a higher number of connections in their concept nodes, but that words with low-imageability and abstract words are connected with a higher amount of different nodes which will cause slower activation and retrieval time.

Since activation of concepts follows a fluid pattern and is dependent on the quality of the connections, highly imageable words will, along with higher activation, require lower cognitive effort. In terms of the spreading activation theory of the connectionist model of semantic memory in the search process of language processing, connections that link the target concept with other concepts are activated. This activation is dependent on the quality and quantity of their connections. Because of that, de Groot (1989) concludes that the activation of highly imageable words will be faster than the activation of low imageability words. The semantic memory is considered as a connectionist model in which each concept is made of concepts and nodes of features connected to other concept nodes. A greater number of activated features results in faster search and activation of the target concepts in healthy subjects. According to the theory of faster automatic spreading of activation in schizophrenia subjects, the more connections to other nodes, i.e. the more features, a concept has, the greater the likeliness that it will activate more competing concepts which need to be inhibited prior to production. Because of this, the stimulus on both tests was made uniform on account of the imageability and frequency conditions, because highly imageable words are expected to have more connections, and highly frequent words are thought to have stronger and more easily activated connections in the semantic memory.

In a language-association production and comprehension study, Sommer et al. (1963) conclude that, although patients with schizophrenia produce more idiosyncratic associations on a production test than a healthy control group, they still choose the most frequent ones on a comprehension test (on the Kent and Rosanoff word-association norms). On account of this, they conclude that patients with schizophrenia can discern frequent from non-frequent associations.

The aim of this study was to determine how two lexical-semantic features, imageability and frequency, affect language-association production and comprehension in subjects with first-episode and early-course schizophrenia-spectrum psychosis. The first task included elicited-association production on stimuli with high or low imageability ratings. The second task was a forced-choice comprehension task which included a frequency condition, as well as an imageability 
condition. In the production test, both groups were expected to have longer reaction times on associations with low imageability in comparison to associations with high imageability. On the comprehension test, both groups were expected to choose statistically more often high- and medium-frequency associations in comparison to low-frequency associations. Additionally, on the comprehension test, the patient group was expected to have longer reaction times in relation to the control group not dependent on the frequency condition. Better understanding of language processing in first-episode and early-course psychosis could enable the creation of diagnostic markers and instruments for predicting the course of the disorder, especially if possibly accompanied with longitudinal studies.

\section{Methods}

\subsection{Participants}

The study included 12 subjects with first-episode and early-course schizophrenia-spectrum psychosis and a control group of 12 matched healthy subjects. The average age of the patient group was 25.29 ( $\mathrm{SD}=3.53$ ) years; 2 patients were right-handed, 4 patients were female, and 8 were male. The average time after the onset of the disorder was 11.88 ( $\mathrm{SD}=14.37)$ months, and the average time after the beginning of therapy was $6.7(\mathrm{SD}=7.4)$ months. All patients received antipsychotic therapy. Their average daily dose of antipsychotics expressed in chlorpromazine equivalents was $600(\mathrm{SD}=298.74) \mathrm{mg}$. The schizophrenia subjects were patients of the University Psychiatric Hospital Vrapče, and the control group were volunteers. The control group was matched with the patient group in terms of age, sex, and dominant hand. All subjects had signed an informed consent for the study, which had been approved by the Ethics Committee of the University Psychiatric Hospital Vrapče on 23 March 2018 (Registry number: 23305/8-18).

\subsection{Materials and procedure}

The study was composed of two tests, an elicited-association production test and a forced-choice comprehension test. Both tests were constructed specifically for this study based on the psycholinguistic literature (Sommer et al., 1963; Rossell \& David, 2005; Kircher et al., 2008). Materials were constructed using lexical items from the Psiholex_HR database (Erdeljac, Sekulić Sović \& Miklić, 2018), which includes, inter alia, data points for associations and imageability collected from native speakers of Croatian or health participants (n:105). On the production test, the subjects were presented with 15 highly imageable words and 15 words with low imageability ratings based on the Psiholex_HR database, on 
which they were to produce associations. Although the subjects were asked to produce three associations for each experimental item, only the first produced association was included in the analysis. On the comprehension test, subjects were also presented with 15 highly imageable words and 15 words with low imageability ratings based on the Psiholex_HR database. After each word, three associations were shown. The associations were also chosen from the the Psiholex_HR database (Erdeljac, Sekulić Sović \& Miklić, 2018). Each item included a highly frequent, moderately frequent and non-frequent association, according to the Psiholex_HR database, from which the subjects were asked to choose the one they thought was most associated with the stimulus. Each experimental item was shown individually, and both the experimental items and the answers were randomized. Both tests were carried out on a computer screen using the E-prime (Schneider et al., 2012) program. Stimulus onset asynchrony was $200 \mathrm{~ms}$, and each stimulus was shown on the screen for one second.

For the production test, reaction times for each experimental item were calculated. Reaction times for words with high and low imageability were compared. For the production test, both the reaction times for the imageability and frequency conditions and the number of chosen associations based on the frequency condition were analyzed.

\section{Results}

\subsection{Production test}

Data for the statistical analysis seems to satisfy conditions for a parametric analysis - Kolmogorov-Smirnov and Shapiro-Wilk tests of normality of results distribution were shown to be non-significant for reaction times of both words with high imageability and those with low imageability, and the same applies for the Levene test of homogenous variances. However, residual distribution was shown to be non-normal in both cases, and when the small number of subjects is taken into account, the validity of these tests is questionable. In small subject samples, neither test has the statistical strength to detect significant differences in normality and homogeneity assumptions of variances (Field, 2013). Therefore, non-parametric analyses were used - the Mann-Whitney U test for group differences and the Wilcoxon signed-rank test for in-group differences. The $p$-value was set at 0.05 . Descriptive data is shown in Table 1. 
Table 1. Descriptive data (median and interquartile range) for reaction times for high-and lowimageability associations in both groups

\begin{tabular}{|l|c|c|c|c|c|c|}
\hline & \multicolumn{3}{|c|}{$\begin{array}{c}\text { Control group } \\
(N=12)\end{array}$} & \multicolumn{3}{c|}{$\begin{array}{c}\text { Patient group } \\
\text { (N= 12) }\end{array}$} \\
\hline & $C$ & Q1 & Q3 & C & Q1 & Q3 \\
\hline Reaction times (ms) & & & & & & \\
\hline $\begin{array}{l}\text { High-imageability } \\
\text { associations }\end{array}$ & 1955.87 & 1763.45 & 2492.73 & 3030.70 & 2641.58 & 3289.33 \\
\hline $\begin{array}{l}\text { Low-imageability } \\
\text { associations }\end{array}$ & 2278.67 & 1957.48 & 2573.77 & 3155.40 & 2839.12 & 3508.73 \\
\hline
\end{tabular}

The Mann-Whitney $\mathrm{U}$ test showed that there is a statistically relevant difference $(U(12,12)=131, z=3.41, p<0.01, r=0.70)$ in average reaction times for high-imageability associations between the control group $(C=1955.87 \mathrm{~ms})$ and the patient group $(C=3030.70 \mathrm{~ms})$. The results were the same for the low-imageability associations, as the average reaction times of the control group $(C=$ $2278.67 \mathrm{~ms})$ and the patient group $(C=3155.40 \mathrm{~ms})$ were statistically relevantly different $(U(12,12)=135, z=3.64, \mathrm{p}<0.01, r=0.74)$.

The Wilcoxon signed-rank test for in-group differences showed that there were statistically relevant differences in reaction time in the control group $(T(12)=66, z=2.12, p=0.03, r=0.43)$, as the control subjects had faster reaction times for high-imageability associations $(C=1955.87 \mathrm{~ms})$ when compared to low-imageability associations $(C=2278.67 \mathrm{~ms})$. However, there were no statistically relevant differences in reaction times with regard to imageability in the control group $(T(12)=50, z=0.86, p=0.39, r=0.18)$. These results can be seen in Figure 1. 


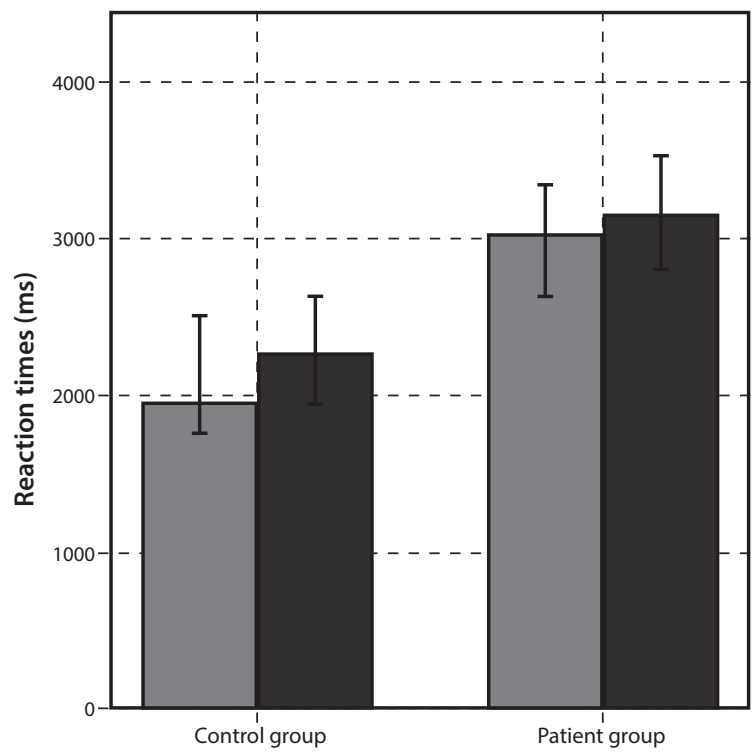

High imageability associations

Low imageability associations

\section{(1)}

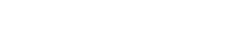

Patient group

Figure 1. Median and reliability intervals of reaction times (ms) for imageability in the control and patient groups

\subsection{Comprehension test}

The data for the statistical analysis deviate from a normal distribution of results and residuals and do not meet the homogeneity of variances condition, and, as in the production test, the subject sample was small. On account of this, non-parametric tests were used - the Mann-Whitney U test for between group differences and the Wilcoxon signed-rank test for in-group differences; Friedman's ANOVA was also used for in-group differences. The $p$-value for all tests was set at 0.05 . With multiple comparisons the Holm-Bonferroni correction was used. Descriptive data can be seen in Table 2.

Table 2. Descriptive data (median and interquartile range) for reaction times for high- and low-imageability associations and reaction times and the number of chosen high-, medium-, and low-frequency associations in both the control and the patient group

\begin{tabular}{|l|c|c|c|c|c|c|}
\hline & \multicolumn{3}{|c|}{$\begin{array}{c}\text { Control group } \\
(N=12)\end{array}$} & \multicolumn{3}{c|}{$\begin{array}{c}\text { Patient group } \\
(N=12)\end{array}$} \\
\hline & $C$ & Q1 & Q3 & C & Q1 & Q3 \\
\hline Reaction times (ms) & & & & & & \\
\hline $\begin{array}{l}\text { High-imageability } \\
\text { associations }\end{array}$ & 1635.20 & 1038.38 & 2026.35 & 2598.23 & 2283.15 & 3052.48 \\
\hline $\begin{array}{l}\text { Low-imageability } \\
\text { associations }\end{array}$ & 1357.10 & 1090.70 & 1839.82 & 2700.23 & 2206.17 & 3072.43 \\
\hline
\end{tabular}




\begin{tabular}{|c|c|c|c|c|c|c|}
\hline & \multicolumn{3}{|c|}{$\begin{array}{l}\text { Control group } \\
\quad(N=12)\end{array}$} & \multicolumn{3}{|c|}{$\begin{array}{l}\text { Patient group } \\
\quad(N=12)\end{array}$} \\
\hline & $C$ & Q1 & Q3 & $C$ & Q1 & Q3 \\
\hline $\begin{array}{l}\text { High-frequency } \\
\text { associations }\end{array}$ & 1278.12 & 1037.39 & 1910.45 & 2275.20 & 2017.97 & 2901.69 \\
\hline $\begin{array}{l}\text { Medium-frequency } \\
\text { associations }\end{array}$ & 1645.82 & 1184.86 & 2143.80 & 2587.99 & 2147.40 & 2983.38 \\
\hline $\begin{array}{l}\text { Low-frequency } \\
\text { associations }\end{array}$ & 2562.89 & 1840.25 & 3070 & 3546.17 & 2225.03 & 4279.63 \\
\hline \multicolumn{7}{|l|}{$\begin{array}{l}\text { Number of chosen } \\
\text { associations }(N)\end{array}$} \\
\hline $\begin{array}{l}\text { High-frequency } \\
\text { associations }\end{array}$ & 21 & 18 & 25 & 22 & 12.50 & 24 \\
\hline $\begin{array}{l}\text { Medium-frequency } \\
\text { associations }\end{array}$ & 8.50 & 5 & 9.75 & 6.50 & 5 & 11.50 \\
\hline $\begin{array}{l}\text { Low-frequency } \\
\text { associations }\end{array}$ & 0.50 & 0 & 2 & 2 & 1 & 5.50 \\
\hline
\end{tabular}

To establish if there were reaction-time differences on high- and low-imageability associations between the two groups, the Mann-Whitney $U$ test was used. It showed that there is a statistically relevant difference $(U(12,12)=126, z=3.12$, $p=0.01, r=0.64)$ in average reaction times on high-imageable associations between the control group ( $C=1635.20 \mathrm{~ms})$ and the patient group ( $C=2598.23 \mathrm{~ms}$ ). The same result was obtained for the reaction times on low-imageability associations, and there was a statistically relevant difference $(U(12,12)=131, z=3.41, \mathrm{p}$ $<0.01, r=0.74)$ on low-imageability associations between the control group ( $C$ $=1357.10 \mathrm{~ms})$ and the patient group $(C=2700.23 \mathrm{~ms})$.

The Wilcoxon signed-rank test showed no statistically relevant differences in reaction times on low- and high-imageability words in the comprehension test in both the control group $(T(12)=60, z=1.65, p=0.09, r=0.34)$ and the patient group $(T(12)=24, z=-1.18, p=0.24, r=-0.24)$. These results can be seen in Figure 2. 


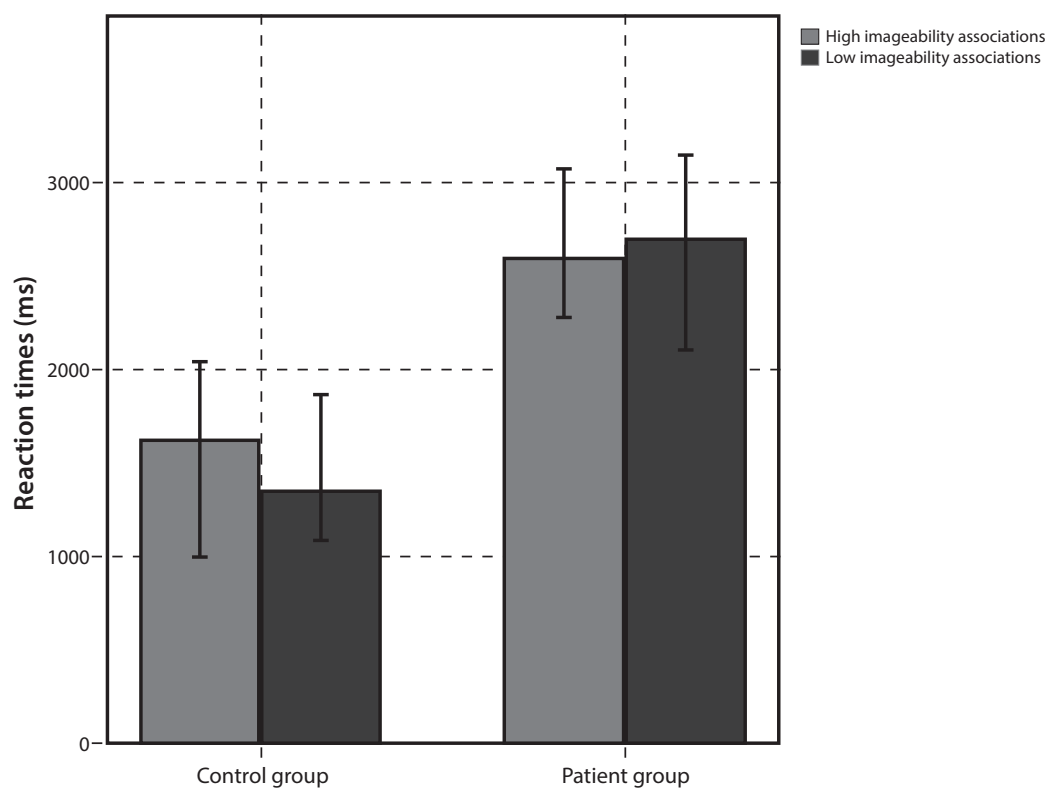

Figure 2. Median and interquartile intervals of reaction-time reliability (ms) for associations' imageabilities in the control and patient groups

To review group differences in reaction times for high-, medium-, and low-frequency associations, the Mann-Whitney $U$ test was used. It showed that there is a statistically relevant difference $(U(12,12)=129, z=3.29, p<0.01, r=0.67)$ in average reaction times on high-frequency associations between the control $(C=1278.12 \mathrm{~ms})$ and patient $(C=2275.20 \mathrm{~ms})$ groups. A statistically relevant difference $(U(12,12)=122, z=2.89, p=0.03, r=0.59)$ was also found in the average reaction times on medium-frequency associations between the control $(C=1645.82 \mathrm{~ms})$ and patient $(C=2587.99 \mathrm{~ms})$ groups. However, the difference was not statistically relevant for low-frequency associations $(U(10,10)=44, z=$ $1.52, p=0.15, r=0.34)$. These results can be seen in Figure 3 . 


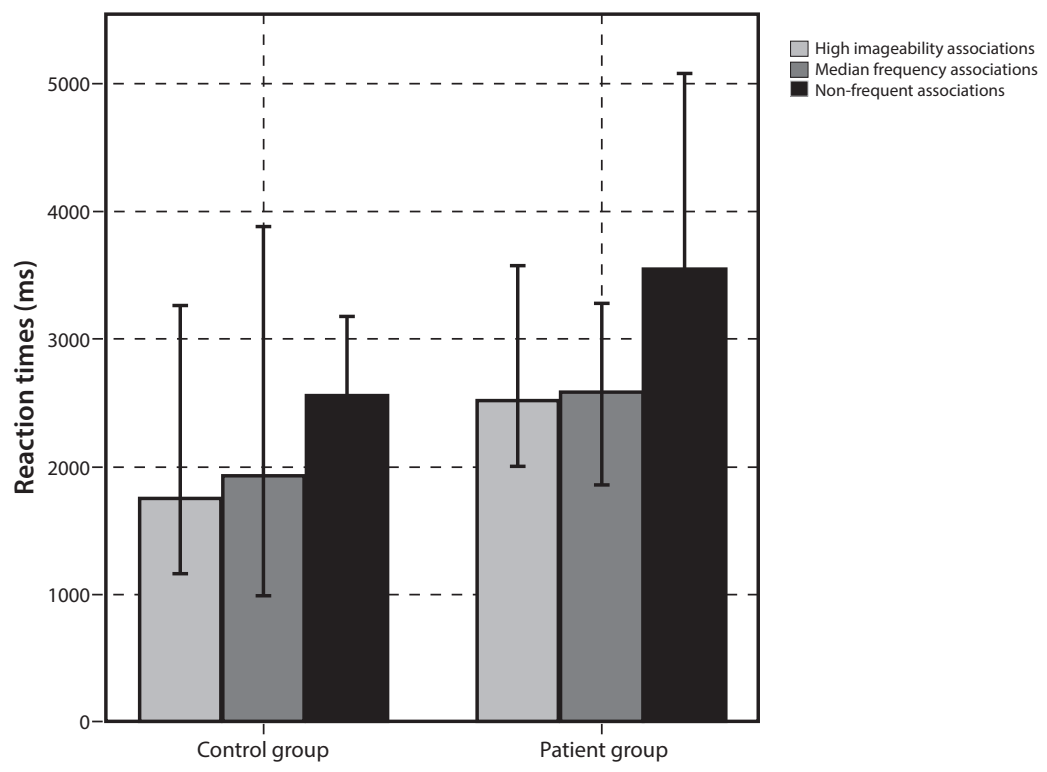

Figure 3. Median and interquartile intervals of reaction-time reliability (ms) for associations' frequencies in the control and patient group

Friedman's ANOVA was used to test in-group differences in reaction times and the number of chosen associations on account of the frequency condition in both groups. In the first analysis, the analysis was shown not to be significant for the control $(\chi 2(2)=2.33, p=0.31)$ or the patient group $(\chi 2(2)=3.80, p=0.15)$, which shows that there were no differences in reaction-time distribution in the frequency condition choices. In the second analysis, the analysis was shown to be significant in both the control $(\chi 2(2)=20.72, p<0.01)$ and the patient group $(\chi 2(2)=16.17, p<0.01)$.

In the control group, pair comparisons (adjusted Wilcoxon tests) show a significant difference $(T(12)=1.83, z=4.49, p<0.01, r=0.92)$ in the average number of highly-frequency associations chosen $(C=21)$ when compared to low-frequent associations $(C=0,50)$, as well as a significant difference $(T(12)=$ $1.04, z=2.55, p=0.03, r=0.52)$ in the average number of median frequency associations chosen $(C=8,50)$ when compared to non-frequent associations. The difference between high- and medium-frequency associations was not shown to be relevant $(T(12)=0.79, z=1.94, p=0.16, r=0.40)$.

In the patient group, pair comparisons have similar results - there is a significant difference $(T(12)=1.58, z=3.88, p<0.01, r=0.79)$ in the average number of high-frequency associations chosen $(C=22)$ when compared to low-frequency associations $(C=2)$. Furthermore, there is a significant difference $(T(12)=$ $1.17, z=2.86, p=0.01, r=0.58)$ in the average number of medium-frequency associations chosen $(C=6.50)$ when compared to low-frequency associations. As 
in the control group, there was no significant difference between high- and mediium-frequency associations $(T(12)=0.42, z=1.02, p=0.92, r=0.21)$. These results can be seen in Figure 4.

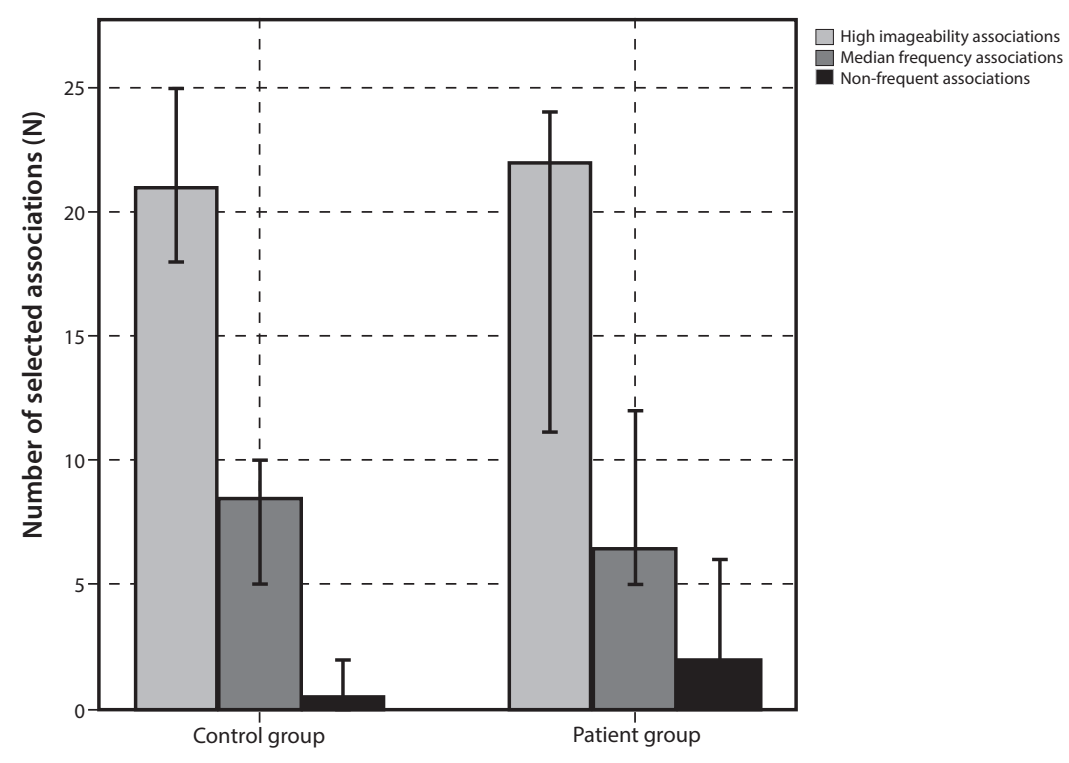

Figure 4. Median and interquartile reliability intervals on the frequency condition in the control and patient groups

\section{Discussion}

The control group had longer reaction times on low-imageability associations, while the patient group reaction times did not statistically differ on the imageability condition. Faster reaction times on highly imageable associations is in line with other studies on imageability processing (Paivio, 1991). The presupposition is that, in schizophrenia patients, highly imageable associations produced a higher amount of activation in the semantic memory and that the decrease in inhibition resulted in longer reaction times, which was the same result as for associations with low-imageability that did not have greater activation in the semantic memory.

Both groups statistically significantly more often chose high- and medium-frequency associations than low-frequency associations. Despite the fact that, on the language production test, the patients with first-episode and early-course psychosis more often produce idiosyncratic, personal, and indirect associations (Maher et al., 2005), on the comprehension test they recognize which ones are most frequently use. 
The patients had longer reaction times than the control group only on associations of high and medium frequency, but not on low-frequency associations. This result is in line with the result of the production test, as the control group had no need for heightened inhibition on non-frequent associations, because they had no need for inhibition on low-imageability associations on the production test, so the reaction times for low-frequency associations did not differ from the reaction times of the control group, while the reaction times for highly and moderately frequent associations were longer than those of the control group.

\section{Conclusion}

Psycholinguistic studies on language production and comprehension contribute to understanding of deficits in psychosis and can contribute to clinical diagnostics. This study analyzed language production and the comprehension of associations in subjects with first-episode and early-course schizophrenia-spectrum psychosis in relation to a healthy control group with regard to the lexical-semantic imageability and frequency features. Our results analysis showed that there is an influence of the imageability feature on language processing in first-episode and early-course psychosis subjects which differs from the influence it has in healthy subjects. In the control subjects, the language stimulus with high imageability facilitated faster processing, but in the patient group, it did not, since more concept features led to higher activation in the semantic memory, which slowed processing as the inhibition mechanisms had to be activated to a higher degree, and it is presupposed that psychosis patients have decreased inhibition together with increased activation in language processing. Similar results were obtained in the reception test for the frequency condition, where the patient group had slower reaction times than the control group on high- and mediumfrequency associations, but not on low-frequency associations.

It can be concluded that psycholinguistic studies on imageability can contribute to the understanding of language processing both in subjects with language disorders and in healthy subjects with normal language processing abilities. A shortcoming of this study is its small subject sample, but further studies will certainly provide great support in creating a complete view of language deficits in patients with schizophrenia and other psychotic disorders. 


\section{References}

Ballerini, M. (2016). Semantic processing and semantic experience in people with schizophrenia: A bridge between phenomenological psychopathology and neuroscience? Journal of Psychopathology, 22, 94-105.

Bokat, C. E., \& Goldberg, T. E. (2003). Letter and category fluency in schizophrenic patients: A meta-analysis. Schizophrenia Research 64, 73-78.

De Groot, A. M. (1989). Representational aspects of word imageability and word frequency as assessed through word association. Journal of Experimental Psychology: Learning, Memory, and Cognition, 15(5), 824-845.

Elvevåg, B., Foltz, P. W., Weinberger, D. R., \& Goldberg, T. E. (2007). Quantifying incoherence in speech: An automated methodology and novel application to schizophrenia. Schizophrenia Research, 93(1-3), 304-316.

Erdeljac, V., Sekulić Sović, M., \& Miklić D. (2018) Psycholingustic Database - Psiholex_ $H R$. Zagreb: Department of Linguistics, Faculty of Humanities and Social Sciences, University of Zagreb.

Field, A. (2013). Discovering statistics using IBM SPSS Statistics (4th ed.). London: SAGE Publications.

Kreher, D. A., Holcomb, P. J., Goff, D., \& Kuperberg, G. R. (2008). Neural evidence for faster and further automatic spreading activation in schizophrenic thought disorder. Schizophrenia Bulletin 34(3), 473-482.

Maher, B. A., Manschreck, T. C., Linner, J., \& Candela, S. (2005). Quantitative assessment of the frequency of normal associations in the utterances of schizophrenia patients and healthy controls. Schizophrenia Research, 78(2-9), 219-224.

Paivio, A. (1991). Dual coding theory: Retrospect and current status. Canadian Journal of Psychology/Revue canadienne de psychologie, 45(3), 255-287.

Schneider, W., Eschman, A., \& Zuccolotto, A. (2012). Using E-prime, 2.0 software, Psychology Software Tools. Pittsburgh: Psychology Software Tools, Inc.

Sommer, R., Dewar, R., \& Osmond, H. (1963). Is there a schizophrenic language? Archives of General Psychiatry, 3, 665-673.

Spitzer, M., Braun, U., Hermle, L., \& Maier, S. (1993). Associative semantic network dysfunction in thought-disordered schizophrenic patients: Direct evidence from indirect semantic priming. Biological Psychiatry 34(12), 864-877. 